Article

\title{
Semi-Preparative Separation of 10 Caffeoylquinic Acid Derivatives Using High Speed Counter-Current Chromatogaphy Combined with Semi-Preparative HPLC from the Roots of Burdock (Arctium lappa L.)
}

\author{
Zhenjia Zheng ${ }^{1}$, Xiao Wang ${ }^{2}$, Pengli Liu ${ }^{1}$, Meng Li ${ }^{1}$, Hongjing Dong ${ }^{2, *}$ and Xuguang Qiao ${ }^{1, *}$ \\ 1 College of Food Science and Engineering, Shandong Agricultural University, Taian 271018, China; \\ pengyou-jia@163.com (Z.Z.); milly.liu@163.com (P.L.); Lemon982436@163.com (M.L.) \\ 2 Key Laboratory of TCM Quality Control Technology, Shandong Analysis and Test Center, Qilu University of \\ Technology (Shandong Academy of Sciences), Jinan 250014, China; wangx@sdas.org \\ * Correspondence: donghongjing_2006@163.com (H.D.); xgqiao@sdau.edu.cn (X.Q.); \\ Tel.: +86-531-8260-5319 (H.D.); +86-538-8242-875 (X.Q.)
}

Received: 28 January 2018; Accepted: 13 February 2018; Published: 15 February 2018

\begin{abstract}
Burdock roots are healthy dietary supplements and a kind of famous traditional Chinese medicine, which contains large amounts of caffeoylquinic acid derivatives. However, little research has been reported on the preparative separation of these compounds from burdock roots. In the present study, a combinative method of HSCCC and semi-preparative HPLC was developed for the semi-preparative separation of caffeoylquinic acid derivatives from the burdock roots. The ethyl acetate extract of burdock roots was first fractionated by MCI macroporous resin chromatography and give three fractions (Fr. 1-3) from the elution of 40\% methanol. Then, these three fractions $(120 \mathrm{mg})$ were separately subjected to HSCCC for purification with the solvent system composed of petroleum ether-ethyl acetate-methanol-water at different volume ratios, and the mixtures were further purified by semi-preparative HPLC. As a result, a total of eight known caffeoylquinic acid derivatives including 3-O-caffeoylquinic acid (32.7 mg, 95.7\%), 1,5-O-dicaffeoylquinic acid (4.3 $\mathrm{mg}, 97.2 \%)$, 3-O-caffeoylquinic acid methyl ester (12.1 mg, 93.2\%), 1,3-O-dicaffeoylquinic acid (42.9 mg, 91.1\%), 1,5-O-dicaffeoyl-3-O-(4-maloyl)-quinic acid ( $4.3 \mathrm{mg}, 84.5 \%$ ), 4,5-O-dicaffeoylquinic acid ( $5.3 \mathrm{mg}, 95.5 \%)$, 1,5-O-dicaffeoyl-3-O-succinylquinic acid $(8.7 \mathrm{mg}, 93.4 \%)$, and 1,5-O-dicaffeoyl-4-O-succinylquinic acid $(1.7 \mathrm{mg}, 91.8 \%)$, and two new compounds were obtained. The new compounds were 1,4-O-dicaffeoyl-3-succinyl methyl ester quinic acid (14.6 mg, 96.1\%) and 1,5-O-dicaffeoyl-3-O-succinyl methyl ester quinic acid $(3.1 \mathrm{mg}, 92.6 \%)$, respectively. The research indicated that the combination of HSCCC and semi-preparative HPLC is a highly efficient approach for preparative separation of the instability and bioactive caffeoylquinic acid derivatives from natural products.
\end{abstract}

Keywords: burdock root; new caffeoylquinic acid derivatives; high speed counter-current chromatography; semi-preparative HPLC

\section{Introduction}

Burdock (Arctium lappa L.) is a biennial plant of the Asteraceae family and its root has been consumed as a tonic vegetable in Asia. Burdock root is not only a healthy and nutritive dietary supplement, it was also a folk herbal medicine with heat-clearing and detoxifying effect [1]. Pharmacological studies indicated that burdock root has antioxidant [2], antiallergic [3], anti-inflammatory [4] and hepatoprotective activities [5]. Caffeoyl quinic acid derivatives are considered to be one of the main active ingredients, such as 1,3-, 1,4-, 1,5-dicaffeoylquinic acids, which were all positional isomers in chemical structure [6,7]. 
Due to the similarity of caffeoylquinic acids, the purification was usually achieved by repeated reverse silica gel column chromatography in the past [8,9]. However, a crude sample is not acceptable when using expensive reverse silica gel, and it usually requires multi-steps of traditional chromatography for the pre-treatment of crude samples. These procedures have the drawbacks of long processing time and low repeatability. Moreover, some compounds with less stability will degrade during the repeat and long separation procedure. Therefore, it is necessary to develop an efficient and repeatable method for the rapid purification of caffeoylquinic acids from natural materials.

High-speed counter-current chromatography (HSCCC) is a continuous liquid-liquid partition chromatography based on partition of compounds between two immiscible liquid phases. It has many advantages such as no irreversible adsorption, low risk of sample denaturation, total sample recovery, and low cost $[10,11]$. This method has been successfully applied to fractionate and purify 3-caffeoylquinic acid and 3,5-, 4,5-dicaffeoylquinic acids from complex natural extracts with high efficiency [12-14]. However, there has been little research until now involving the purification of 1,3, 1,4-, 1,5-caffeoylquinic acids by HSCCC [15].

In this work, the crude extract of burdock roots was fractionated into three fractions by MCI macroporous resin. HSCCC was developed for the purification and separation of caffeoylquinic acid derivatives from these three fractions, and the compounds with low purity were further purified by semi-preparative HPLC. Finally, 10 isomers of caffeoyl quinic acid derivatives were obtained, among which two were new (compounds I, J). The structure of these compounds is shown in Figure 1.

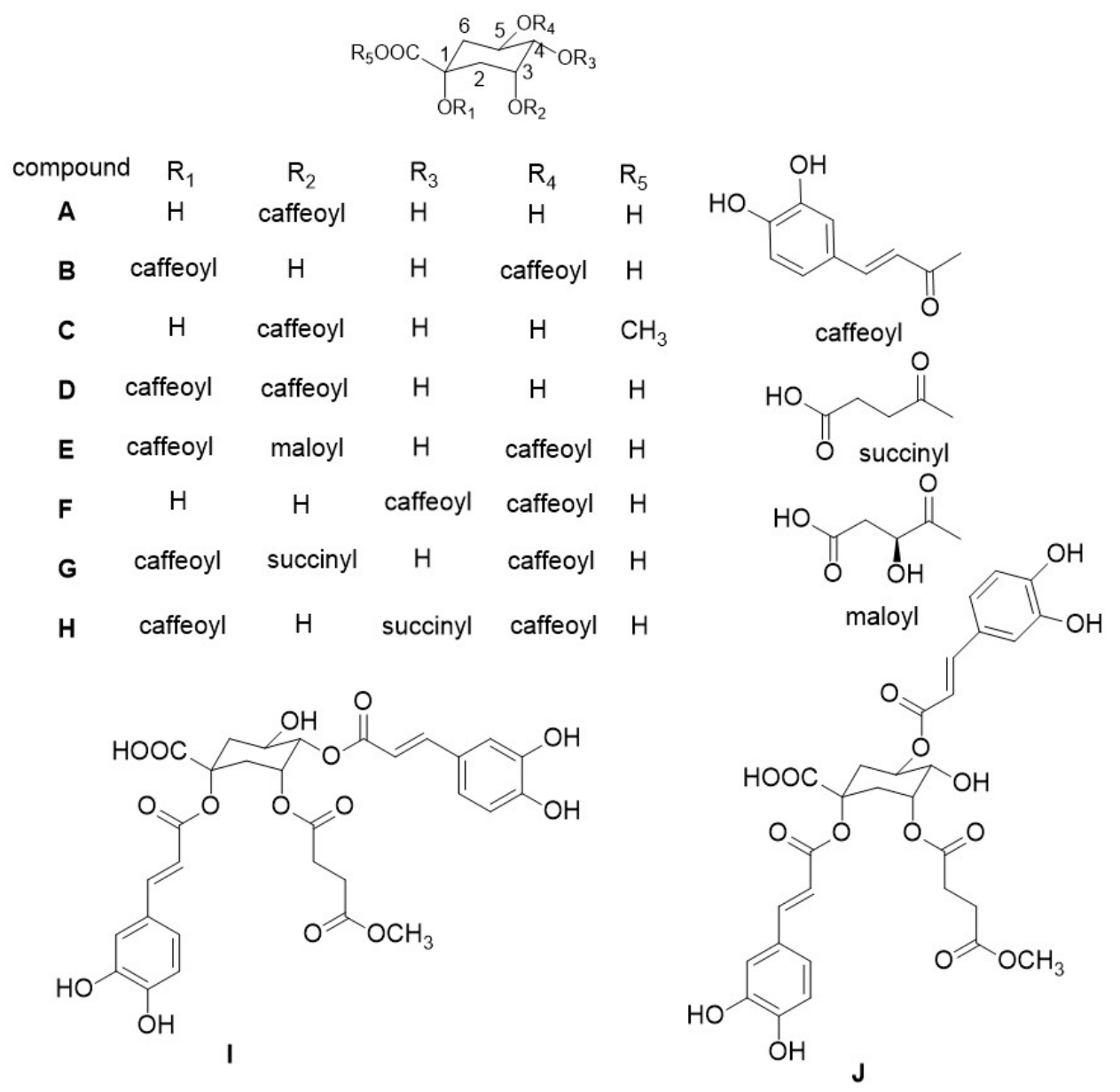

Figure 1. Chemical structures of ten caffeoylquinic acid derivatives from burdock roots. 


\section{Results and Discussion}

\subsection{Optimization of HSCCC Conditions}

The search of appropriate solvent system plays an important role in separation of HSCCC. Suitable two-phase solvent system should satisfy several principles, such as the good solubility, high retention of the stationary phase, and the most important was the suitable partition coefficients $\left(K_{\mathrm{D}}\right)$ for target compounds and separation factors between adjacent peaks $[10,16]$. To achieve a successful separation using HSCCC, according to the purification of 3,5-, 4,5-dicaffeoylquinic acids using HSCCC by wang et al. [14], and polarity, solubility of caffeoylquinic acid derivatives, the $K_{D}$ values of these 10 compounds in the solvent system composed of Pet-EtOAc-MeOH-water were measured and are given in Table 1.

Table 1. The $K_{\mathrm{D}}$ values of caffeoylquinic acid derivatives from burdock roots.

\begin{tabular}{|c|c|c|c|c|c|c|c|c|c|c|}
\hline \multirow{2}{*}{ Sample } & \multirow{2}{*}{$\begin{array}{l}\text { Solvent System (Pet-EtOAc- } \\
\left.\mathrm{MeOH}-\mathrm{H}_{2} \mathrm{O}, v / v\right)\end{array}$} & \multicolumn{9}{|c|}{$K_{\mathrm{D}}$} \\
\hline & & A & B & $C$ & D & $\mathrm{E}$ & $\mathbf{F}$ & G & $\mathbf{H}$ & $\mathrm{I} / \mathrm{J}$ \\
\hline \multirow[b]{2}{*}{ Fr. 1} & 0:5:0:5 & 0.55 & 0.58 & 4.82 & & & & & & \\
\hline & 0:5:0.5:5 & 0.47 & 0.52 & 2.21 & & & & & & \\
\hline \multirow{3}{*}{ Fr. 2} & $0: 5: 1: 5$ & & & & 26.88 & & & & & \\
\hline & 1:5:1:5 & & & & 4.00 & & & & & \\
\hline & 1:4:1:4 & & & & 2.03 & & & & & \\
\hline \multirow{2}{*}{ Fr. 3} & 1:4:1:4 & & & & & 0.50 & 2.16 & 2.29 & 2.25 & 7.88 \\
\hline & 1:4:2:3 & & & & & 0.19 & 0.29 & 0.39 & 0.41 & 0.76 \\
\hline
\end{tabular}

\subsubsection{Separation of Fr. 1 by HSCCC}

As shown in Table 1, the solvent system composed of EtOAc-MeOH-water $(5: 0.5: 5, v / v)$ provided suitable $K_{\mathrm{D}}$ value for compound $\mathbf{C}$ (2.21). However, the $K_{\mathrm{D}}$ values for compounds $\mathbf{A}$ and $\mathbf{B}$ were close to each other, which may result in the partially overlap of compounds $\mathbf{A}$ and $\mathbf{B}$ in HSCCC separation. In order to achieve good separation of compounds $\mathbf{A}$ and $\mathbf{B}$, methanol was removed to reduce the elution ability of the mobile phase and improve the resolution, and the $K_{\mathrm{D}}$ values of compounds $\mathbf{A}-\mathbf{C}$ were measured in the solvent system composed of EtOAc-water (5:5, v/v). From Table 1, it can be seen that EtOAc-water $(5: 5, v / v)$ still provided close $K_{\mathrm{D}}$ values for compounds $\mathbf{A}$ and $\mathbf{B}(0.55,0.58)$ and larger $K_{\mathrm{D}}$ values for compound $\mathbf{C}$ (4.82), and thus the separation of compounds $\mathbf{A}$ and $\mathbf{B}$ may be very difficult using this solvent system. Considering the suitable $K_{\mathrm{D}}$ values and good separation factor of compound $\mathrm{C}$ in the solvent system composed of EtOAc-MeOH-water (5:0.5:5, v/v), the solvent system was used for the separation of Fr. 1 (Figure 2). When the two-phase solvent system was applied, compounds A (28.5 mg, peak A) and C (12.1 mg, peak C) were obtained with purities of $95.7 \%$ and 93.2\%, respectively, from $120 \mathrm{mg}$ of Fr.1 (Figure S2), and compound B was eluted together as a mixture (15.6 mg) with small portion of compound A after analysis by HPLC.

The mixture $(15.6 \mathrm{mg})$ was further purified by semi-preparative HPLC with the solvent of acetonitrile-water containing $0.1 \% \mathrm{HCOOH}(25: 75, v / v)$. Finally, compounds $\mathbf{A}$ and $\mathbf{B}$ can be largely isolated from the mixture, and obtained $4.2 \mathrm{mg}$ of compound A and $4.3 \mathrm{mg}$ of compound $\mathbf{B}$ with purities of $97.9 \%$ and $97.2 \%$ (Figure S2), respectively.

\subsubsection{Separation of Fr. 2 by HSCCC}

According to the separation of Fr. 1 by HSCCC and polarity of compound $\mathbf{D}$ in Fr. 2, the $K_{\mathrm{D}}$ values of compound $\mathbf{D}$ in the solvent system composed of EtOAc-MeOH-water (5:1:5, v/v) was measured, and the $K_{\mathrm{D}}$ value (26.88) was too large that it will consume a large amount of solvent to elute compound $\mathbf{D}$. By adding a small amount of Pet and increasing the ratio of $\mathrm{MeOH}$, the $K_{\mathrm{D}}$ values changed to be smaller (Table 1). The solvent system composed of Pet-EtOAc-MeOH-water (1:4:1:4,v/v) provided 
suitable $K_{\mathrm{D}}$ values for compound $\mathbf{D}$ (2.03). When this solvent system was used for the purification (Figure 2), $42.9 \mathrm{mg}$ of compound $\mathbf{D}$ was obtained with purity of $91.1 \%$ from $120 \mathrm{mg}$ of Fr. 2 (Figure S3).

\subsubsection{Separation of Fr. 3 by HSCCC}

As shown in Table 1, the solvent system composed of Pet-EtOAc-MeOH-water (1:4:1:4, $v / v)$ provided suitable $K_{\mathrm{D}}$ values for compounds $\mathbf{E}-\mathbf{I}(0.50,2.10,2.21,3.47,7.88)$. However, the $K_{\mathrm{D}}$ values of compounds $\mathrm{F}$ and $\mathrm{G}$ were very close $(2.10,2.21)$, and the two compounds would be eluted together in the HSCCC separation. Figure 2 shows the HSCCC chromatography for the separation of Fr. 3 using the solvent system composed of Pet-EtOH-MeOH-water (1:4:1:4, $v / v)$, and five peaks were obtained. After analysis by HPLC, peaks E, H-J all contain only one compounds. Though the compounds of peak $\mathbf{I}$ and $\mathbf{J}$ have the same elution time in HPLC, they were two different compounds, which were named compound I and J, separately. After being collected and dried, $4.3 \mathrm{mg}$ of compound $\mathbf{E}, 1.7 \mathrm{mg}$ of compound $\mathbf{H}, 14.6 \mathrm{mg}$ of compound $\mathbf{I}$, and $3.1 \mathrm{mg}$ of compound $\mathbf{J}$ were obtained from $120 \mathrm{mg}$ of Fr. 3. The purities of these compounds were all determined by peak area normalization method at $280 \mathrm{~nm}$, and they were $84.5 \%, 91.8 \%, 96.1 \%$, and $92.6 \%$ (Figure S4), respectively.

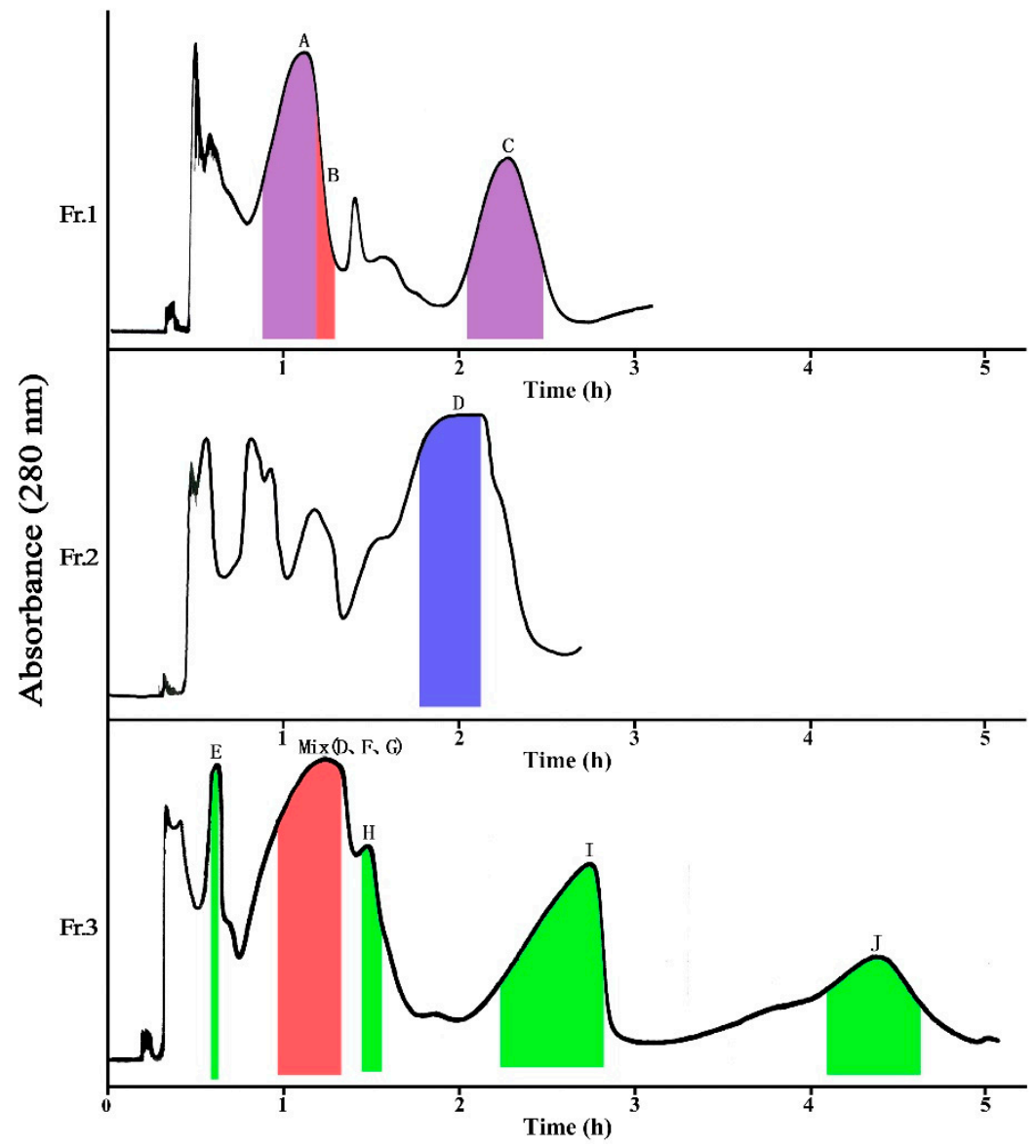

Figure 2. High-speed counter-current chromatography (HSCCC) chromatograms of Fr. 1-3.

Compounds $\mathbf{F}$ and $\mathbf{G}$ were eluted as a mixture. The mixture was further purified by semi-preparative HPLC with methanol-water containing $0.1 \% \mathrm{HCOOH}(25: 75, v / v)$. As a result, $5.3 \mathrm{mg}$ of compound $\mathbf{F}$ and $8.7 \mathrm{mg}$ of compound $\mathbf{G}$ were obtained from $39.8 \mathrm{mg}$ of mixture with purity of $95.5 \%$ and $93.4 \%$ (Figure S4), respectively.

In all, six purified compounds were obtained after one-step separation of HSCCC and semi-preparative HPLC separation from Fr. 3. 
Fr. 1 Solvent systems: Pet-EtOAc-MeOH-H $\mathrm{H}_{2} \mathrm{O}(0: 5: 0.5: 5, v / v)$; Fr. 2 Solvent systems: Pet-EtOAc-MeOH-H ${ }_{2} \mathrm{O}(1: 4: 1: 4, v / v)$; Fr. 3 solvent system: Pet-EtOAc-MeOH-H ${ }_{2} \mathrm{O}(1: 4: 1: 4, v / v)$. sample size: $120 \mathrm{mg}$; flow-rate: $5.0 \mathrm{~mL} / \mathrm{min}$; detection: $280 \mathrm{~nm}$.

\subsection{Identification of Compounds}

\subsubsection{Identification of Known Compounds}

Compound A ESI-MS, $m / z$ 355.1035 $[\mathrm{M}+\mathrm{H}]^{+}, 353.0890[\mathrm{M}-\mathrm{H}]^{-},{ }^{1} \mathrm{H}-\mathrm{NMR}\left(\mathrm{DMSO}-d_{6}, 400 \mathrm{MHz}\right)$ $\delta$ (ppm): 1.76-2.03 (4H, m, H-2, 6), 5.08 (1H, m, H-3), 3.58 (1H, m, H-4), 3.93 (1H, m, H-5), 7.04 (1H, br s, H-2' $), 6.77\left(1 \mathrm{H}, \mathrm{d}, J=8.0 \mathrm{~Hz}, \mathrm{H}-5^{\prime}\right), 6.98\left(1 \mathrm{H}, \mathrm{d}, J=7.8 \mathrm{~Hz}, \mathrm{H}-6^{\prime}\right), 7.42\left(1 \mathrm{H}, \mathrm{d}, J=16.0 \mathrm{~Hz}, \mathrm{H}-7^{\prime}\right), 6.15(1 \mathrm{H}$, $\left.\mathrm{d}, J=16.0 \mathrm{~Hz}, \mathrm{H}-8^{\prime}\right) ;{ }^{13} \mathrm{C}-\mathrm{NMR}\left(\mathrm{DMSO}-\mathrm{d}_{6}, 100 \mathrm{MHz}\right) \delta(\mathrm{ppm}): 73.6(\mathrm{C}-1), 37.2(\mathrm{C}-2), 68.3(\mathrm{C}-3), 70.5$ (C-4), 70.9 (C-5), 37.2 (C-6), $175.0(\mathrm{C}-7), 125.6\left(\mathrm{C}-1^{\prime}\right), 114.8\left(\mathrm{C}-2^{\prime}\right), 145.6\left(\mathrm{C}-3^{\prime}\right), 148.3\left(\mathrm{C}-4^{\prime}\right), 115.8\left(\mathrm{C}-5^{\prime}\right)$, $121.4\left(\mathrm{C}-6^{\prime}\right), 144.9\left(\mathrm{C}-7^{\prime}\right), 114.3\left(\mathrm{C}-8^{\prime}\right), 165.8\left(\mathrm{C}-9^{\prime}\right)$. Compared with reported data [17], compound A was identified as 3-O-caffeoylquinic acid.

Compound B ESI-MS, $m / z$ 517.1313 $[\mathrm{M}+\mathrm{H}]^{+}, 515.1202[\mathrm{M}-\mathrm{H}]^{-},{ }^{1} \mathrm{H}-\mathrm{NMR}\left(\mathrm{DMSO}-d_{6}, 400 \mathrm{MHz}\right)$ $\delta$ (ppm): 1.76-2.47 (4H, m, H-2, 6), 3.98 (1H, m, H-3), 3.50 (1H, m, H-4), 5.28 (1H, m, H-5), 7.01, 6.91 (each $1 \mathrm{H}$, br s, H-2' , $2^{\prime \prime}$ ), 6.67, 6.53 (each $\left.1 \mathrm{H}, \mathrm{d}, J=8.0 \mathrm{~Hz}, \mathrm{H}-5^{\prime}, 5^{\prime \prime}\right), 6.87,6.63$ (each $1 \mathrm{H}, \mathrm{d}, J=8.0 \mathrm{~Hz}, \mathrm{H}-6^{\prime}$, $\left.6^{\prime \prime}\right), 7.42,7.40$ (each $\left.1 \mathrm{H}, \mathrm{d}, J=16.0 \mathrm{~Hz}, \mathrm{H}-7^{\prime}, 7^{\prime \prime}\right), 6.20,6.07$ (each $\left.1 \mathrm{H}, \mathrm{d}, J=16.0 \mathrm{~Hz}, \mathrm{H}-8^{\prime}, 8^{\prime \prime}\right) ;{ }^{13} \mathrm{C}-\mathrm{NMR}$ $\left(\mathrm{DMSO}-d_{6}, 100 \mathrm{MHz}\right) \delta(\mathrm{ppm}): 80.1$ (C-1), 34.2 (C-2), 66.5 (C-3), 73.2 (C-4), 71.4 (C-5), 39.4 (C-6), 173.1 (C-7), 125.7, $125.8\left(\mathrm{C}-1^{\prime}, 1^{\prime \prime}\right), 116.2,115.7\left(\mathrm{C}-2^{\prime}, 2^{\prime \prime}\right), 145.3,146.0\left(\mathrm{C}-3^{\prime}, 3^{\prime \prime}\right), 148.6,148.9\left(\mathrm{C}-4^{\prime}, 4^{\prime \prime}\right), 116.4$, $116.3\left(\mathrm{C}-5^{\prime}, 5^{\prime \prime}\right), 120.5,121.4\left(\mathrm{C}-6^{\prime}, 6^{\prime \prime}\right), 145.8,145.8\left(\mathrm{C}-7^{\prime}, 7^{\prime \prime}\right), 114.6,114.9\left(\mathrm{C}-8^{\prime}, 8^{\prime \prime}\right), 166.5,165.7\left(\mathrm{C}-9^{\prime}\right.$, $\left.9^{\prime \prime}\right)$. Compared with reported data [18], compound B was identified as 1,5-O-dicaffeoylquinic acid.

Compound C EIS-MS, $m / z$ 369.1518 $[\mathrm{M}+\mathrm{H}]^{+}, 367.1044[\mathrm{M}-\mathrm{H}]^{-} ;{ }^{1} \mathrm{H}-\mathrm{NMR}\left(400 \mathrm{MHz}, \mathrm{DMSO}-d_{6}\right)$ $\delta$ (ppm): $2.12(2 \mathrm{H}, \mathrm{m}, \mathrm{H}-2 \mathrm{e}, 6 \mathrm{a}), 1.77(1 \mathrm{H}, \mathrm{dd}, J=9.6,12.4 \mathrm{~Hz}, \mathrm{H}-2 \mathrm{a}), 5.02(1 \mathrm{H}, \mathrm{d}, J=3.6 \mathrm{~Hz}, \mathrm{H}-3)$, $3.57(1 \mathrm{H}, \mathrm{m}, \mathrm{H}-4), 3.88(1 \mathrm{H}, \mathrm{d}, J=8.8 \mathrm{~Hz}, \mathrm{H}-5), 1.93(1 \mathrm{H}, \mathrm{dd}, J=13.6,3.2 \mathrm{~Hz}, \mathrm{H}-6 \mathrm{e}), 7.03\left(1 \mathrm{H}, \mathrm{s}, \mathrm{H}-2^{\prime}\right)$, $6.78\left(1 \mathrm{H}, \mathrm{d}, J=8.0 \mathrm{~Hz}, \mathrm{H}-5^{\prime}\right), 6.98\left(1 \mathrm{H}, \mathrm{d}, J=8.0 \mathrm{~Hz}, \mathrm{H}-6^{\prime}\right), 7.39\left(1 \mathrm{H}, \mathrm{d}, J=16.0 \mathrm{~Hz}, \mathrm{H}-7^{\prime}\right), 6.11(1 \mathrm{H}, \mathrm{d}$, $\left.J=16.0 \mathrm{~Hz}, \mathrm{H}-8^{\prime}\right), 3.58\left(3 \mathrm{H}, \mathrm{s},-\mathrm{OCH}_{3}\right) ;{ }^{13} \mathrm{C}-\mathrm{NMR}\left(100 \mathrm{MHz}, \mathrm{DMSO}-d_{6}\right) \delta(\mathrm{ppm}): 73.5(\mathrm{C}-1), 35.6(\mathrm{C}-2)$, $71.5(\mathrm{C}-3), 69.8(\mathrm{C}-4), 67.3(\mathrm{C}-5), 37.7(\mathrm{C}-6), 125.8\left(\mathrm{C}-1^{\prime}\right), 115.1\left(\mathrm{C}-2^{\prime}\right), 145.6\left(\mathrm{C}-3^{\prime}\right), 148.9\left(\mathrm{C}-4^{\prime}\right), 116.3$ $\left(\mathrm{C}-5^{\prime}\right), 121.8\left(\mathrm{C}-6^{\prime}\right), 146.08\left(\mathrm{C}-7^{\prime}\right), 114.3\left(\mathrm{C}-8^{\prime}\right), 165.8\left(\mathrm{C}-9^{\prime}\right), 52.3\left(-\mathrm{OCH}_{3}\right), 174.1(\mathrm{C}-7)$. Compared with reported data [19], compound $\mathbf{C}$ was identified as 3-O-Caffeoylquinic acid methyl ester.

Compound D ESI-MS, $m / z 517.1360[\mathrm{M}+\mathrm{H}]^{+}, 515.1258[\mathrm{M}-\mathrm{H}]^{-}{ }^{1}{ }^{1} \mathrm{H}-\mathrm{NMR}\left(\mathrm{DMSO}-d_{6}, 400 \mathrm{MHz}\right)$ $\delta$ (ppm): $1.91(1 \mathrm{H}, \mathrm{dd}, J=9.2,13.2 \mathrm{~Hz}, \mathrm{H}-2), 2.32(1 \mathrm{H}, \mathrm{dd}, J=10.4,13.2 \mathrm{~Hz}, \mathrm{H}-2), 5.23(1 \mathrm{H}, \mathrm{m}, \mathrm{H}-3)$, $3.62(1 \mathrm{H}, \mathrm{d}, J=6.0 \mathrm{~Hz}, \mathrm{H}-4), 4.08$ (1H, br s, H-5), 2.28 (2H, br s, H-6), $7.05(1 \mathrm{H}$, br s, H-2') , 6.78 (1H, d, $\left.J=8.0 \mathrm{~Hz}, \mathrm{H}-5^{\prime}\right), 7.00\left(1 \mathrm{H}, \mathrm{d}, J=8.0 \mathrm{~Hz}, \mathrm{H}-6^{\prime}\right), 7.48\left(1 \mathrm{H}, \mathrm{d}, J=16.0 \mathrm{~Hz}, \mathrm{H}-7^{\prime}\right), 6.21(1 \mathrm{H}, \mathrm{d}, J=16.0 \mathrm{~Hz}$, H-8 $\left.{ }^{\prime}\right)$, 7. $05\left(1 \mathrm{H}\right.$, br s, H-2 $\left.{ }^{\prime \prime}\right), 6.78\left(1 \mathrm{H}, \mathrm{d}, J=7.2 \mathrm{~Hz}, \mathrm{H}-5^{\prime \prime}\right), 7.00\left(1 \mathrm{H}, \mathrm{d}, J=8.0 \mathrm{~Hz}, \mathrm{H}-6^{\prime \prime}\right), 7.47(1 \mathrm{H}$, $\left.\mathrm{d}, J=15.8 \mathrm{~Hz}, \mathrm{H}-7^{\prime \prime}\right), 6.21\left(1 \mathrm{H}, \mathrm{d}, J=16.0 \mathrm{~Hz}, \mathrm{H}-8^{\prime \prime}\right) ;{ }^{13} \mathrm{C}-\mathrm{NMR}\left(\mathrm{DMSO}-d_{6}, 100 \mathrm{MHz}\right) \delta$ (ppm): 79.9 (C-1), 36.3 (C-2), 70.5 (C-3), 71.4 (C-4), 68.0 (C-5), 34.6 (C-6), $126.0\left(\mathrm{C}-1^{\prime}\right), 115.3\left(\mathrm{C}-2^{\prime}\right), 146.1\left(\mathrm{C}-3^{\prime}\right), 148.9$ $\left(\mathrm{C}-4^{\prime}\right), 116.3\left(\mathrm{C}-5^{\prime}\right), 121.8\left(\mathrm{C}-6^{\prime}\right), 145.8\left(\mathrm{C}-7^{\prime}\right), 114.9\left(\mathrm{C}-8^{\prime}\right), 166.5\left(\mathrm{C}-9^{\prime}\right), 126.0\left(\mathrm{C}-1^{\prime \prime}\right), 115.3\left(\mathrm{C}-2^{\prime \prime}\right), 146.1$ $\left(\mathrm{C}-3^{\prime \prime}\right), 148.9\left(\mathrm{C}-4^{\prime \prime}\right), 116.3\left(\mathrm{C}-5^{\prime \prime}\right), 121.7\left(\mathrm{C}-6^{\prime \prime}\right), 145.6\left(\mathrm{C}-7^{\prime \prime}\right), 114.7\left(\mathrm{C}-8^{\prime \prime}\right), 165.7\left(\mathrm{C}-9^{\prime \prime}\right), 173.1(-\mathrm{COOH})$. Compared with reported data [20], compound $\mathbf{D}$ was identified as 1, 3-O-dicaffeoylquinic acid.

Compound E ESI-MS, $m / z 633.1460[\mathrm{M}+\mathrm{H}]^{+}, 631.1320[\mathrm{M}-\mathrm{H}]^{-} ;{ }^{1} \mathrm{H}-\mathrm{NMR}\left(\mathrm{DMSO}-d_{6}, 400 \mathrm{MHz}\right)$ $\delta$ (ppm): 2.41-2.67 (2H, m, overlap, H-2), 5.30 (1H, m, H-3), $3.86(1 \mathrm{H}, \mathrm{dd}, J=3.2,8.4 \mathrm{~Hz}, \mathrm{H}-4), 5.19(1 \mathrm{H}$, m, H-5), 1.98 (1H, m, H-6), 2.47 (1H, m, H-6, overlap), $4.27\left(1 \mathrm{H}, \mathrm{m}, \mathrm{H}-2^{\prime}\right), 2.41-2.67\left(2 \mathrm{H}, \mathrm{m}, \mathrm{H}-3^{\prime}\right), 7.01$, 7.06 (each $1 \mathrm{H}$, br s, H-2' , 2') 6.78 (each $1 \mathrm{H}, \mathrm{d}, J=8.0 \mathrm{~Hz}, \mathrm{H}-5^{\prime}, 5^{\prime \prime}$ ), 7.01 (each $1 \mathrm{H}, \mathrm{d}, J=8.0 \mathrm{~Hz}, \mathrm{H}-6^{\prime}$, $\left.6^{\prime \prime}\right), 7.50,7.50$ (each $\left.1 \mathrm{H}, \mathrm{d}, J=15.6 \mathrm{~Hz}, \mathrm{H}-7^{\prime}, 7^{\prime \prime}\right), 6.27,6.23\left(\right.$ each $\left.1 \mathrm{H}, \mathrm{d}, J=15.6 \mathrm{~Hz}, \mathrm{H}-8^{\prime}, 8^{\prime \prime}\right)$; ${ }^{13} \mathrm{C}-\mathrm{NMR}$ $\left(\mathrm{DMSO}-d_{6}, 100 \mathrm{MHz}\right) \delta(\mathrm{ppm}): 79.2$ (C-1), 32.3 (C-2), 71.6 (C-3), 69.0 (C-4), 70.2 (C-5), 35.8 (C-6), 172.4 (C-7), $174.9\left(\mathrm{C}-1^{\prime}\right), 67.2\left(\mathrm{C}-2^{\prime}\right), 39.7$ (C-3', overlap), $170.2\left(\mathrm{C}-4^{\prime}\right), 125.9,126.0\left(\mathrm{C}-1^{\prime \prime}, 1^{\prime \prime \prime}\right), 115.3,115.5$ $\left(\mathrm{C}-2^{\prime \prime}, 2^{\prime \prime \prime}\right), 145.9,146.5\left(\mathrm{C}-3^{\prime \prime}, 3^{\prime \prime \prime}\right), 148.9,149.0\left(\mathrm{C}-4^{\prime \prime}, 4^{\prime \prime \prime}\right), 116.3,116.3\left(\mathrm{C}-5^{\prime \prime}, 5^{\prime \prime \prime}\right), 121.8,121.9\left(\mathrm{C}-6^{\prime \prime}\right.$, 
$\left.6^{\prime \prime \prime}\right), 146.1,146.1\left(\mathrm{C}-7^{\prime \prime}, 7^{\prime \prime \prime}\right), 114.2,114.4\left(\mathrm{C}-8^{\prime \prime}, 8^{\prime \prime \prime}\right), 165.7,166.4\left(\mathrm{C}-9^{\prime \prime}, 9^{\prime \prime \prime}\right)$. Compared with reported data [21], compound $\mathbf{E}$ was identified as 1,5-O-dicaffeoyl-3-O-(4-maloyl)-quinic acid.

Compound F ESI-MS, $m / z 517.1334[\mathrm{M}+\mathrm{H}]^{+}, 515.1212[\mathrm{M}-\mathrm{H}]^{-} ;{ }^{1} \mathrm{H}-\mathrm{NMR}\left(\mathrm{CD}_{3} \mathrm{OD}, 400 \mathrm{MHz}\right)$ $\delta$ (ppm): 2.09-2.32 (4H, m, H-2, 6), $4.38(1 \mathrm{H}, \mathrm{m}, \mathrm{H}-3), 5.13(1 \mathrm{H}, \mathrm{dd}, J=3.2,9.2 \mathrm{~Hz}, \mathrm{H}-4), 5.62(1 \mathrm{H}, \mathrm{m}$, H-5), 7.01, 7.03 (each 1H, H-2' , 2") , 6.74, 6.76 (each 1H, d, J = 8.0 Hz, H-5' , 5' ), 6.90, 6.92 (each 1H, dd, $\left.J=2.4,8.0 \mathrm{~Hz}, \mathrm{H}-6^{\prime}, 6^{\prime \prime}\right), 6.19,6.29$ (each $\left.1 \mathrm{H}, \mathrm{d}, J=16.0 \mathrm{~Hz}, \mathrm{H}-8^{\prime}, 8^{\prime \prime}\right), 7.52,7.60$ (each $1 \mathrm{H}, \mathrm{d}, J=16.0 \mathrm{~Hz}$ ). ${ }^{13} \mathrm{C}-\mathrm{NMR}\left(\mathrm{CD}_{3} \mathrm{OD}, 100 \mathrm{MHz}\right) \delta(\mathrm{ppm}): 76.1$ (C-1), 38.4 (C-2), 69.4 (C-3), 75.8 (C-4), 69.0 (C-5), 39.4 (C-6), $176.9(\mathrm{C}-7), 127.6,127.7\left(\mathrm{C}-1^{\prime}, 1^{\prime \prime}\right), 115.2,115.2\left(\mathrm{C}-2^{\prime}, 2^{\prime \prime}\right), 146.8\left(\mathrm{C}-3^{\prime}, 3^{\prime \prime}\right), 149.7\left(\mathrm{C}-4^{\prime}, 4^{\prime \prime}\right), 116.5\left(\mathrm{C}-5^{\prime}, 5^{\prime \prime}\right)$, 123.1, 123.1 (C-6', 6" $)$, 147.6, $147.7\left(C-7^{\prime}, 7^{\prime \prime}\right), 114.7,114.8\left(\mathrm{C}-8^{\prime}, 8^{\prime \prime}\right), 168.3,168.6\left(\mathrm{C}-9^{\prime}, 9^{\prime \prime}\right)$. Compared with reported data [22], compound $\mathbf{F}$ was identified as 4, 5-O-dicaffeoyl-quinic acid.

Compound G ESI-MS, $m / z$ 617.1516 [M + H] ${ }^{+}, 615.1369[\mathrm{M}-\mathrm{H}]^{-} ;{ }^{1} \mathrm{H}-\mathrm{NMR}$ (DMSO- $d_{6}, 400 \mathrm{MHz}$ ) $\delta$ (ppm): 2.39-2.55 (2H, m, H-2, overlap), 5. 25 (1H, m, H-3), 3. $84(1 \mathrm{H}, \mathrm{d}, J=8.8 \mathrm{~Hz}, \mathrm{H}-4), 5.22(1 \mathrm{H}$, m, H-5 ), 1.92 (1H, m, H-6), 2.39-2.54 (1H, m, H-6, overlap), 2.39-2.54 (4H, m, H-2' , H-3', overlap), 7.07, 7.07 (each $1 \mathrm{H}$, br s, H-2 $\left.{ }^{\prime \prime}, 2^{\prime \prime \prime}\right), 6.78,6.78$ (each $\left.1 \mathrm{H}, \mathrm{d}, J=8.0 \mathrm{~Hz}, \mathrm{H}-5^{\prime \prime}, 5^{\prime \prime \prime}\right), 7.01,7.01$ (each $1 \mathrm{H}, \mathrm{d}$, $\left.J=8.0 \mathrm{~Hz}, \mathrm{H}-6^{\prime \prime}, 6^{\prime \prime \prime}\right) 7.49,7.50$ (each $\left.1 \mathrm{H}, \mathrm{d}, J=16.0 \mathrm{~Hz}, \mathrm{H}-7^{\prime \prime}, 7^{\prime \prime \prime}\right), 6.25,6.27$ (each 1H, d, $J=16.0 \mathrm{~Hz}$, H-8 ${ }^{\prime \prime}, 8^{\prime \prime \prime}$ ). ${ }^{13} \mathrm{C}-\mathrm{NMR}$ (DMSO- $\left.d_{6}, 100 \mathrm{MHz}\right) \delta$ (ppm): 80.0 (C-1), 32.3 (C-2), 70.3 (C-3), 71.9 (C-4), 69.6 (C-5), 36.6 (C-6), $173.7(\mathrm{C}-7), 172.7\left(\mathrm{C}-1^{\prime}\right), 29.4\left(\mathrm{C}-2^{\prime}\right), 29.2\left(\mathrm{C}-3^{\prime}\right), 172.1\left(\mathrm{C}-4^{\prime}\right), 125.9,126.0\left(\mathrm{C}-1^{\prime \prime}, 1^{\prime \prime \prime}\right)$, 115.4, 115.4 (C-2", $\left.2^{\prime \prime \prime}\right), 146.1,146.1\left(\mathrm{C}-3^{\prime \prime}, 3^{\prime \prime \prime}\right), 149.0,149.0\left(\mathrm{C}-4^{\prime \prime}, 4^{\prime \prime \prime}\right), 116.3,116.4\left(\mathrm{C}-5^{\prime \prime}, 5^{\prime \prime \prime}\right), 122.6$, $122.7\left(\mathrm{C}-6^{\prime \prime}, 6^{\prime \prime \prime}\right), 145.8,145.8\left(\mathrm{C}-7^{\prime \prime}, 7^{\prime \prime \prime}\right), 114.5,114.6\left(\mathrm{C}-8^{\prime \prime}, 8^{\prime \prime \prime}\right), 166.5,165.6\left(\mathrm{C}-9^{\prime \prime}, 9^{\prime \prime \prime}\right)$. Compared with reported data [2], compound $\mathrm{G}$ was identified as 1,5-O-dicaffeoyl-3-O-succinylquinic acid.

Compound H ESI-MS, $m / z 617.1529[\mathrm{M}+\mathrm{H}]^{+}, 615.1374[\mathrm{M}-\mathrm{H}]^{-} ;{ }^{1} \mathrm{H}-\mathrm{NMR}$ (DMSO- $d_{6}, 400 \mathrm{MHz}$ ) $\delta$ (ppm): 2.32-2.60 (2H, m, overlap), $4.24(1 \mathrm{H}, \mathrm{m}, \mathrm{H}-3), 4.92(1 \mathrm{H}, \mathrm{m}), 5.46(1 \mathrm{H}, \mathrm{m}), 2.02-2.07,2.32-2.60$

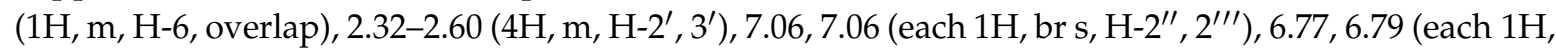
$\left.\mathrm{d}, J=8.0 \mathrm{~Hz}, \mathrm{H}-5^{\prime \prime}, 5^{\prime \prime \prime}\right), 7.01,7.01$ (each $\left.1 \mathrm{H}, \mathrm{d}, J=8.0 \mathrm{~Hz}, \mathrm{H}-6^{\prime \prime}, 6^{\prime \prime \prime}\right), 7.47,7.48$ (each1H, d, $J=15.6 \mathrm{~Hz}$, H-7", $\left.7^{\prime \prime \prime}\right), 6.20,6.24$ (each $\left.1 \mathrm{H}, \mathrm{d}, J=15.6 \mathrm{~Hz}, \mathrm{H}-8^{\prime \prime}, 8^{\prime \prime \prime}\right) ;{ }^{13} \mathrm{C}-\mathrm{NMR}$ (DMSO- $\left.d_{6}, 100 \mathrm{MHz}\right) \delta$ (ppm): 79.5 (C-1), 34.7 (C-2), 65.6 (C-3), 74.4 (C-4), 67.2 (C-5), 36.8 (C-6), 173.8 (C-7), 172.2 (C-1'), 29.4 (C-2'), $29.2\left(\mathrm{C}-3^{\prime}\right), 172.1\left(\mathrm{C}-4^{\prime}\right), 126.0,126.0\left(\mathrm{C}-1^{\prime \prime}, 1^{\prime \prime \prime}\right), 115.3,115.4\left(\mathrm{C}-2^{\prime \prime}, 2^{\prime \prime \prime}\right), 146.1,146.1\left(\mathrm{C}-3^{\prime \prime}, 3^{\prime \prime \prime}\right), 148.9$, $149.0\left(\mathrm{C}-4^{\prime \prime}, 4^{\prime \prime \prime}\right), 116.3,116.4\left(\mathrm{C}-5^{\prime \prime}, 5^{\prime \prime \prime}\right), 121.7,121.9\left(\mathrm{C}-6^{\prime \prime}, 6^{\prime \prime \prime}\right), 146.1,146.1\left(\mathrm{C}-7^{\prime \prime}, 7^{\prime \prime \prime}\right), 114.0,114.8$ $\left(\mathrm{C}-8^{\prime \prime}, 8^{\prime \prime \prime}\right), 165.7,166.2\left(\mathrm{C}-9^{\prime \prime}, 9^{\prime \prime \prime}\right)$. Compared with reported data [2], compound $\mathbf{H}$ was identified as 1-,5-O-dicaffeoyl-4-O-succinylquinic acid.

\subsubsection{Identification of New Compounds}

Compound I was isolated as a white amorphous powder. HR-ESI-MS spectrum gave a molecular ion peak at $\mathrm{m} / \mathrm{z} 631.16532[\mathrm{M}+\mathrm{H}]^{+}$(calc.631.16575), indicating a molecular formula of $\mathrm{C}_{30} \mathrm{H}_{30} \mathrm{O}_{15} \cdot{ }^{1} \mathrm{H}-\mathrm{NMR}$ and ${ }^{13} \mathrm{C}-\mathrm{NMR}$ spectra exhibited four doublets with coupling constants of $15.6 \mathrm{~Hz}$ characteristic for trans olefinic protons $\left(\delta_{\mathrm{H}} 6.23,6.31,7.47,7.50\right)$. The coupling pattern of six aromatic proton signals $(7.06,7.07$ (each 1H, br s), 6.66, 6.78 (each 1H, d, 8.4 Hz), 7.00, 7.03 (each 1H, d, 8.4 Hz)) appearing as two $\mathrm{ABX}$ systems in ${ }^{1} \mathrm{H}_{-}{ }^{1} \mathrm{H}$ COSY (Figure 3) spectrum, which indicated the presence two caffeic acid moieties [22]. The ${ }^{1} \mathrm{H}-\mathrm{NMR}$ signals at $\delta_{\mathrm{H}} 2.31-2.47(4 \mathrm{H}, \mathrm{m})$ and ${ }^{13} \mathrm{C}-\mathrm{NMR}$ signals at $\delta_{\mathrm{C}}$ 28.8, 29.3, 172.4, 172.6 indicated the presence of succinyl moiety. The ${ }^{1} \mathrm{H}-\mathrm{NMR}$ signals at $\delta_{\mathrm{H}} 3.48(3 \mathrm{H}, \mathrm{s})$ and ${ }^{13} \mathrm{C}-\mathrm{NMR}$ signals at $\delta_{\mathrm{C}} 51.8$ indicated the presence of methoxyl moiety. The rest of the signals of ${ }^{1} \mathrm{H}-\mathrm{NMR}$ and ${ }^{13} \mathrm{C}-\mathrm{NMR}$ were attributed to a quinic acid moiety. The low-filed signals of $\delta_{\mathrm{H}} 5.38(1 \mathrm{H}, \mathrm{m}$, $\mathrm{H}-3)$, and $\delta_{\mathrm{H}} 4.88(1 \mathrm{H}, \mathrm{dd}, 3.2 \mathrm{~Hz}, 8.8 \mathrm{~Hz})$ indicated the substituted of $3-\mathrm{OH}$ and $4-\mathrm{OH}$, the low-filed of $\delta_{\mathrm{C}} 79.7$ indicated the substituted of 1-OH. Therefore, compound I was a caffeoyl quinic acid derivatives with three acyl groups at 1,3 , and 5 positions of quinic acid.

The location of the two caffeoyl groups and one succinyl group on the quinic acid moiety was deduced by HMBC spectrum (Figure 3). The correlation of $\delta_{\mathrm{H}} 5.38(1 \mathrm{H}, \mathrm{m}, \mathrm{H}-3)$ and $\delta_{\mathrm{H}} 4.88(1 \mathrm{H}$, dd, $3.2 \mathrm{~Hz}, 8.8 \mathrm{~Hz}$ ) to $171.4,166.5$, separately, which indicated the substitution of a caffeoyl group on $\mathrm{C}-4$, a succinyl group on C-3. Moreover, the correlation of $\delta_{\mathrm{H}} 3.48(3 \mathrm{H}, \mathrm{s})$ to $\delta_{\mathrm{C}} 172.4$ indicated the 
esterification of succinic acid. Conclusively, the other caffeoyl group was unambiguously connected to 1-OH. Therefore, the compound was identified as 1,4-O-dicaffeoyl-3-succinyl methyl ester quinic acid. The detail ${ }^{1} \mathrm{H}-\mathrm{NMR}$ and ${ }^{13} \mathrm{C}-\mathrm{NMR}$ data were listed in Table 2.

Compound $\mathbf{J}$ was isolated as a white amorphous powder. HR-ESI-MS spectrum gave a molecular ion peak at $\mathrm{m} / \mathrm{z} 631.16814[\mathrm{M}+\mathrm{H}]^{+}$(calc.631.16575), indicating a molecular formula of $\mathrm{C}_{30} \mathrm{H}_{30} \mathrm{O}_{15}$. The molecular ion peak was at $m / z 631.1640[\mathrm{M}+\mathrm{H}]^{+} .{ }^{1} \mathrm{H}-\mathrm{NMR}$ and ${ }^{13} \mathrm{C}-\mathrm{NMR}$ spectra were similar with compound I and also exhibited two caffeic acid moieties, one succinyl moiety, and one $-\mathrm{OCH}_{3}$ (Table 2), which indicated that compound $\mathbf{J}$ was also a caffeoylquinic acid derivatives with three acyl groups. The low-filed signals of $\delta_{\mathrm{H}} 5.26(1 \mathrm{H}, \mathrm{m}, \mathrm{H}-3)$, and $\delta_{\mathrm{H}} 5.23(1 \mathrm{H}, \mathrm{m})$ indicated the substituted of $3-\mathrm{OH}$ and $5-\mathrm{OH}$, and the low-filed of $\delta_{\mathrm{C}} 79.7$ indicated the substituted of 1-OH. These data were similar with 1,5-O-dicaffeoyl-3-O-succinylquinic acid as reported [22], and the differences were the presence of one $-\mathrm{OCH}_{3}$.

Table 2. ${ }^{1} \mathrm{H}-\mathrm{NMR}(400 \mathrm{MHz})$ and ${ }^{13} \mathrm{C}-\mathrm{NMR}(100 \mathrm{MHz})$ data of compound I and $\mathbf{J}\left(\right.$ DMSO- $\left.d_{6}\right)$.

\begin{tabular}{|c|c|c|c|c|}
\hline \multirow[t]{2}{*}{ Position } & \multicolumn{2}{|l|}{ Compound I } & \multicolumn{2}{|l|}{ Compound J } \\
\hline & $\delta_{\mathrm{H}}(\mathrm{ppm}, \mathrm{Mult}, J$ in $\mathrm{Hz})$ & $\delta_{\mathrm{C}}(\mathrm{ppm})$ & $\delta_{\mathrm{H}}(\mathrm{ppm}$, Mult, $J$ in $\mathrm{Hz})$ & $\begin{array}{c}\delta_{\mathrm{C}} \\
(\mathrm{ppm})\end{array}$ \\
\hline 1 & & 79.7 & & 78.8 \\
\hline 2 & 2.31-2.47 (m, overlap) & 32.3 & 2.34-2.58 (m, overlap) & 31.4 \\
\hline 3 & $5.38(\mathrm{~d}, 3.6)$ & 69.1 & $5.26(\mathrm{~m})$ & 69.5 \\
\hline 4 & $4.88(\mathrm{dd}, 3.2,8.8)$ & 74.7 & $3.85(\mathrm{dd}, 3.2,8.8)$ & 71.3 \\
\hline 5 & $4.14(\mathrm{~m})$ & 63.9 & $5.23(\mathrm{~m})$ & 68.7 \\
\hline 6 & $1.92(\mathrm{~m}), 2.31-2.47$ (m, overlap) & 39.4 (overlap) & $1.93(\mathrm{~m}), 2.34-2.58(\mathrm{~m}$, overlap $)$ & 35.9 \\
\hline 7 & & 172.6 & & 172.0 \\
\hline $1^{\prime}$ & & 171.4 & & 171.7 \\
\hline $2^{\prime}$ & 2.31-2.47 (m, overlap) & 28.8 & 2.34-2.58 (m, overlap) & 28.3 \\
\hline $3^{\prime}$ & $2.31-2.47$ (m, overlap) & $\begin{array}{l}20.0 \\
29.3\end{array}$ & $2.34-2.58(\mathrm{~m}$, overlap) & 28.8 \\
\hline $4^{\prime}$ & & 172.4 & & 171.2 \\
\hline$-\mathrm{OCH}_{3}$ & $3.48(\mathrm{~s})$ & 51.8 & $3.51(\mathrm{~s})$ & 51.3 \\
\hline $1^{\prime \prime}$ & & 126.0 & & 125.3 \\
\hline $2^{\prime \prime}$ & $7.07(1 \mathrm{H}, \mathrm{br} \mathrm{s})$ & 115.4 & 7. 07 (br s) & 114.7 \\
\hline $3^{\prime \prime}$ & & 145.6 (overlap) & & 145.8 \\
\hline $4^{\prime \prime}$ & & 148.9 & & 148.2 \\
\hline $5^{\prime \prime}$ & $6.78(\mathrm{~d}, 8.4)$ & 116.3 & $6.79(\mathrm{~d}, 8.0)$ & 115.7 \\
\hline $6^{\prime \prime}$ & $7.03(\mathrm{~d}, 8.4)$ & 121.9 & 7. $03(\mathrm{dd}, 8.0)$ & 121.3 \\
\hline $7^{\prime \prime}$ & $7.50(\mathrm{~d}, 15.6)$ & 146.1 & $7.51(\mathrm{~d}, 16.0)$ & 145.2 \\
\hline $8^{\prime \prime}$ & $6.31(\mathrm{~d}, 15.6)$ & 114.5 & $6.25(\mathrm{~d}, 16.0)$ & 113.7 \\
\hline $9^{\prime \prime}$ & & 166.5 & & 165.1 \\
\hline $1^{\prime \prime \prime}$ & & 125.9 & & 125.4 \\
\hline $2^{\prime \prime \prime}$ & 7.06 (br s) & 115.3 & 7. 07 (br s) & 114.8 \\
\hline $3^{\prime \prime \prime}$ & & 145.6 (overlap) & & 145.8 \\
\hline $4^{\prime \prime \prime}$ & & 149.0 & & 148.3 \\
\hline $5^{\prime \prime \prime}$ & $6.77(\mathrm{~d}, 8.4)$ & 116.2 & $6.80(\mathrm{~d}, 8.0)$ & 115.7 \\
\hline $6^{\prime \prime \prime}$ & $7.00(\mathrm{~d}, 8.4)$ & 121.8 & $7.05(\mathrm{dd}, 8.0)$ & 121.3 \\
\hline $7^{\prime \prime \prime}$ & $7.47(\mathrm{~d}, 15.6)$ & 146.1 & 7. $52(\mathrm{~d}, 16.0)$ & 145.3 \\
\hline $8^{\prime \prime \prime}$ & $6.23(\mathrm{~d}, 15.6)$ & 114.5 & 6. $29(\mathrm{~d}, 16.0)$ & 114.0 \\
\hline $9^{\prime \prime \prime}$ & & 165.8 & & 165.9 \\
\hline
\end{tabular}

The location of the - $\mathrm{OCH}_{3}$ was deduced by HMBC spectrum (Figure 3). The correlation of $\delta_{\mathrm{H}} 3.51$ $(3 \mathrm{H}, \mathrm{s})$ and $\delta_{\mathrm{C}} 171.7$ indicated-OCH $\mathrm{O}_{3}$ was located in succiny quinic acid. Therefore, compound $\mathbf{J}$ was identified as 1,5-O-dicaffeoyl-3-O-succinyl methyl ester quinic acid. 

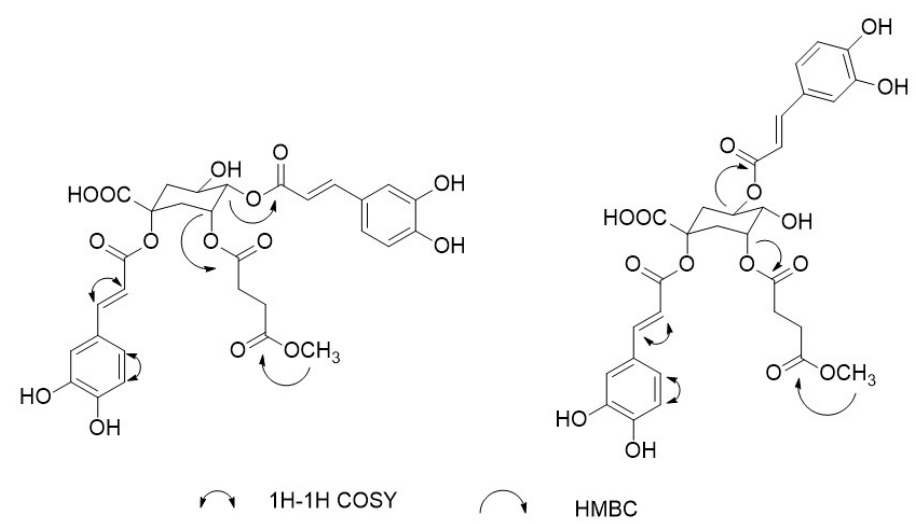

Figure 3. Key $\mathrm{HMBC}$ and ${ }^{1} \mathrm{H}-{ }^{1} \mathrm{H}$ COSY correlations of new compounds.

\section{Experimental}

\subsection{Material and Reagents}

The solvents used in this experiment, including petroleum ether (Pet, 60-90 ${ }^{\circ} \mathrm{C}$ ), ethyl acetate (EtOAc), methanol $(\mathrm{MeOH})$, and 95\% ethanol were all of analytical grade (Tianjin Fuyu Fine Chemical Co., Ltd, Tianjin, China). Methanol and acetonitrile used for HPLC analysis were of chromatographic grade (Oceanpak Alexative Chemical, Ltd, Gothenburg, Sweden). The purified water was prepared by an osmosis Milli-Q system (Millipore, Bedford, MA, USA). Burdock root was purchased from Yiwei Food Co. Ltd. (Pei Town, Jiangsu Province, China) and identified as the roots of Arctium lappa L. by Xiao Wang (Shandong analysis and Test Center, Qilu University of Technology, Shandong Academy of Sciences, Jinan, China). The voucher specimen (No. sdatc-2017-023) was deposited at Shandong analysis and test center.

\subsection{Apparatus}

HSCCC separation was carried out using a TBE-300C instrument (Tauto Biotech, Shanghai, China) with $300 \mathrm{~mL}$ of multilayer coil (I.D. $1.9 \mathrm{~mm}$ ) and a $20 \mathrm{~mL}$ sample loop. The revolution speed was regulated in the range of 0-1000 rpm. The HSCCC system was equipped with a DC-0506 constant temperature circulating device (Tauto Biotech, Shanghai, China) to stabilize the separation temperature. A TBP-5002 constant flow pump was used to pump the solvents. Continuous monitoring of the effluent was achieved using an 8823B-UV detector (Beijing BINTA Instrument Technology Co., Ltd., Beijing, China) at $280 \mathrm{~nm}$. A Model 3057-11 portable recorder (Chongqing Sichuan Instrument Automation Co., Ltd. Chongqing, China) was used to record the chromatograms.

The analysis of all samples was performed using a Waters Alliance system including a Waters 2998 Photodiode Array Detection system, a Waters 2695 Multi-Solvent delivery system, a Waters 2695 system controller, a Waters 2695 pump, and an Empower 3 Workstation (Waters, Milford, MA, USA).

\subsection{Pre-Processing of Crude Sample}

Powdered burdock roots $(1.0 \mathrm{~kg}$ ) was extracted three times with $10 \mathrm{~L}$ of $95 \%$ ethanol under reflux ( $2 \mathrm{~h}$ for each). After filtration, the solvent was concentrated in vacuo at $45{ }^{\circ} \mathrm{C}$ to afford a residue $(83 \mathrm{~g})$. The residue was dissolved in $1000 \mathrm{~mL}$ of water and partitioned with equal volumes of Pet and EtOAc in a separator funnel (each for three times) successively. The EtOAc layers were combined and evaporated under reduced pressure at $45^{\circ} \mathrm{C}$ giving $11.12 \mathrm{~g}$ of EtOAc extract. The EtOAc extract was further separated by MCI column chromatography $(4 \times 40 \mathrm{~cm})$ and eluted with $10 \%(0.9 \mathrm{~L}), 40 \%(2 \mathrm{~L})$, $70 \%(1.65 \mathrm{~L})$, and 100\% (0.55 L) methanol, and 40\% elution solvent was fractionated into Fr. $1(1.12 \mathrm{~g})$, Fr. 2 (3.61 g) and Fr. 3 (0.8 g) according to HPLC analysis (Figure S1). 


\subsection{Optimization of HPLC Conditions}

The ethyl acetate extract of burdock roots was analyzed by HPLC. The mobile phases including methanol/acetonitrile-water containing $0.1 \% \mathrm{HCOOH}$ and column including Phenomenex Gemini-NX C18 $(250 \mathrm{~mm} \times 4.6 \mathrm{~mm}, 5 \mu \mathrm{m})$, YMC-Pack ODS-A $(250 \mathrm{~mm} \times 4.6 \mathrm{~mm}, 5 \mu \mathrm{m})$, Waters Symmetry C18 $(250 \mathrm{~mm} \times 4.6 \mathrm{~mm}, 5 \mu \mathrm{m})$, Spax Technologies Inc. Amethyst C18-H $(250 \mathrm{~mm} \times 4.6 \mathrm{~mm}, 5 \mu \mathrm{m})$ were tested for separation. The results showed that the baseline separation of the target compounds were obtained when the mobile phase was acetonitrile (A)-water containing $0.1 \% \mathrm{HCOOH} \mathrm{(B)}(0-7 \mathrm{~min}, 15 \%$ A; 7-8 $\mathrm{min}, 15-20 \% \mathbf{A} ; 8-29 \mathrm{~min}, 20 \%$ A; $29-30 \mathrm{~min}, 20-25 \% \mathbf{A} ; 30-50 \mathrm{~min}, 25-30 \% \mathbf{A})$, and the column was Phenomenex Gemini-NX C18 (250 mm $\times 4.6 \mathrm{~mm}, 5 \mu \mathrm{m}, 110 \AA$ A $)$. The flow-rate was $1.0 \mathrm{ml} / \mathrm{min}$, and the effluent was monitored at $280 \mathrm{~nm}$ by a DAD detector.

The HPLC chromatography of crude extract and fractionated samples (Fr. 1-3) by MCI macroporous resin is shown in Figure 2, which revealed that Fr. 1 mainly contains compounds A-C, Fr. 2 mainly contains compound D, and Fr. 3 mainly contains compounds E-J.

\subsection{Selection and Preparation of the Two-Phase Solvent System}

The selection of a two-phase solvent system with suitable partition coefficient $\left(K_{\mathrm{D}}\right)$ was very important in the entire work of HSCCC separation. The $K_{\mathrm{D}}$ value was measured as follows: about $2.0 \mathrm{mg}$ of crude sample was added to a test tube, to which $2 \mathrm{~mL}$ of each-phase solvent were added. The test tube was shaken vigorously to promote the dissolve of the samples. After the equilibration was established, both the upper phase and lower phase were analyzed by HPLC. The $K_{\mathrm{D}}$ value was defined as the peak area of target compounds in the stationary phase divided by that in the mobile phase [16].

The designate solvent was poured into a separatory funnel and shaken rapidly. After thoroughly equilibrated at room temperature, the two phases were separated for HSCCC separation.

\subsection{Separation Procedure}

For each separation, the separation column was first filled with the upper phase (stationary phase) at a flow rate of $20.0 \mathrm{~mL} / \mathrm{min}$. Then, the apparatus was rotated forward at $800 \mathrm{rpm}$, while the lower phase was pumped through the column as mobile phase at a flow-rate of $5.0 \mathrm{~mL} / \mathrm{min}$. After the hydrodynamic equilibrium system was established, the sample solution (120 $\mathrm{mg}$ of sample dissolved in $4 \mathrm{~mL}$ of lower phase and $4 \mathrm{~mL}$ of upper phase) was injected via the sample port. The separation process was kept at $25^{\circ} \mathrm{C}$. The effluents were continuously monitored with a UV detector at $280 \mathrm{~nm}$ and collected manually according to the profile of HSCCC chromatography.

\subsection{Semi-Preparative HPLC Separations}

Semi-preparative HPLC separations were operated at YMC C18 $(10.0 \mathrm{~mm} \times 250 \mathrm{~mm}, 5 \mu \mathrm{m})$ column using the solvent composed of acetonitrile-water containing $0.1 \% \mathrm{HCOOH}(25: 75, v / v)$ and methanol-water containing $0.1 \% \mathrm{HCOOH}(25: 75, v / v)$ at a flow-rate of $3.0 \mathrm{~mL} / \mathrm{min}$ and monitored at $280 \mathrm{~nm}$.

\subsection{Identification of Compounds}

The chemical structures of all compounds were determined by 1D, 2D-NMR spectrometry performed on a Bruker AV-400 spectrometer (Bruker BioSpin, Rheinstetten, Germany). HR-ESI-MS experiments were performed on an Agilent 6520 Q-TOF MS (Agilent, Santa Clara, CA, USA).

\section{Conclusions}

Caffeoylquinic acid derivatives are polyphenols that have abundant isomers with varieties of bioactivities. Due to the similarity of these compounds in chemical structure, repeat and multiple chromatography has often been adopted for their purification, which leads to the degrade and loss 
of these compounds. In this manuscript, a total of 10 caffeoylquinic acid derivatives including two new ones were obtained by one-step combination of HSCCC and semi-preparative HPLC. The results demonstrated that the combination of HSCCC with semi-preparative HPLC is a highly efficient means for preparative separation of the non-stable caffeoylquinic acid derivatives from natural products.

Supplementary Materials: The following are available online. HPLC chromatograms of crude extract and purified compounds; NMR, MS spectrums of new compounds.

Acknowledgments: This work is supported by the Special Fund for Agro-Scientific Fesearch in the Public Interest (grant No. 201503142), the Shandong Province Taishan Scholar \{rogram (Lanping Guo), and the Priority Research Program of the Shandong Academy of Sciences (Lanping Guo).

Author Contributions: Zhenjia Zheng conducted the experiment and wrote the paper; Xiao Wang, Pengli Liu, and Meng Li conducted the experiment; Hongjing Dong and Xuguang Qiao designed and guided the experiment.

Conflicts of Interest: The authors declare no conflict of interest.

\section{References}

1. Tian, X.; Sui, S.; Huang, J.; Bai, J.P.; Ren, T.S.; Zhao, Q.C. Neuroprotective effects of Arctium lappa L. roots against glutamate-induced oxidative stress by inhibiting phosphorylation of p38, JNK and ERK 1/2 MAPKs in PC12 cells. Environ. Toxicol. Pharmacol. 2014, 38, 189-198. [CrossRef] [PubMed]

2. Maruta, Y.; Kawabata, J.; Niki, R. Antioxidative caffeoylquinic acid derivatives in the roots of Burdock (Arctium lappa L.). J. Agric. Food Chem. 1995, 43, 2592-2595. [CrossRef]

3. Yang, W.S.; Lee, S.R.; Yong, J.J.; Park, D.W.; Cho, Y.M.; Joo, H.M.; Kim, I.; Seu, Y.B.; Sohn, E.H.; Kang, S.C. Antiallergic Activity of Ethanol Extracts of Arctium lappa L. Undried Roots and Its Active Compound, Oleamide, in Regulating FceRI-Mediated and MAPK Signaling in RBL-2H3 Cells. J. Agric. Food Chem. 2016, 64, 3564-3573. [CrossRef] [PubMed]

4. Lin, C.C.; Lu, J.M.; Yang, J.J.; Chuang, S.C.; Ujiie, T. Anti-inflammatory and radical scavenge effects of Arctium lappa. Am. J. Chin. Med. 1996, 24, 127-137. [CrossRef] [PubMed]

5. Lin, S.C.; Lin, C.H.; Lin, C.C.; Lin, Y.H.; Chen, C.F.; Chen, I.C.; Wang, L.Y. Hepatoprotective effects of Arctium lappa Linne on liver injuries induced by chronic ethanol consumption and potentiated by carbon tetrachloride. J. Biomed. Sci. 2002, 9, 401-409. [CrossRef] [PubMed]

6. Jaiswal, R.; Kuhnert, N. Identification and characterization of five new classes of chlorogenic acids in burdock (Arctium lappa L.) roots by liquid chromatography/tandem mass spectrometry. Food Funct. 2011, 2, 63-71. [CrossRef] [PubMed]

7. Zhao, Y.; Zhao, J.; LI, X.P.; Zhou, C.X.; Sun, H.D.; Hao, X.J.; Xiao, P.P. Advances in caffeoylquinic acid research. China J. Chin. Mater. Med. 2006, 31, 869-874.

8. Jiang, X.W.; Bai, J.P.; Zhang, Q.; Hu, X.L.; Tian, X.; Zhu, J.; Liu, J.; Meng, W.H.; Zhao, Q.C. Caffeoylquinic acid derivatives from the roots of Arctium lappa, L. (burdock) and their structure-activity relationships (SARs) of free radical scavenging activities. Phytochem. Lett. 2016, 15, 159-163. [CrossRef]

9. Eunju, L.; Jusun, K.; Hyunpyo, K.; Jehyun, L.; Kang, S.S. Phenolic constituents from the flower buds of Lonicera japonica and their 5-lipoxygenase inhibitory activities. Food Chem. 2010, 120, 134-139.

10. Ito, Y. Golden rules and pitfalls in selecting optimum conditions for high-speed counter-current chromatography. J. Chromatogr. A 2005, 1065, 145-168. [CrossRef] [PubMed]

11. Skalickawoźniak, K.; Garrard, I. A comprehensive classification of solvent systems used for natural product purifications in countercurrent and centrifugal partition chromatography. Nat. Prod. Rep. 2015, 32, 1556-1561. [CrossRef] [PubMed]

12. Costa, F.D.N.; Jerz, G.; Figueiredo, F.D.S.; Winterhalter, P.; Leitao, G.G. Solvent system selectivities in counter current chromatography using Salicornia gaudichaudiana metabolites as practical example withoff-line electrospray mass-spectrometry injection profiling. J. Chromatogr. A 2015, 1385, 20-27. [CrossRef] [PubMed]

13. Li, A.F.; Zhang, Y.Q.; Sun, A.L.; Liu, R.M. Preparative isolation and purification of phenolic acids from the dried buds of lonicera japonica thumb by high-speed counter-current chromatography in gradient elution mode. J. Liq. Chromatogr. Relat. Technol. 2012, 35, 1933-1944. 
14. Wang, Y.F.; Liu, B. Preparative isolation and purification of dicaffeoylquinic acids from the Ainsliaea fragrans champ by high-speed counter-current chromatography. Phytochem. Anal. 2007, 18, 436-440. [CrossRef] [PubMed]

15. Paek, J.H.; Lim, S.S. Preparative isolation of aldose reductase inhibitory compounds from Nardostachys chinensis by elution-extrusion counter-current chromatography. Arch. Pharm. Res. 2014, 37, 1271-1279. [CrossRef] [PubMed]

16. Zeng, H.L.; Liu, Q.; Yu, J.G.; Jiang, X.Y.; Wu, Z.L.; Wang, M.L.; Chen, M.; Chen, X.Q. One-step separation of nine structural analogues from Poria cocos (Schw.) Wolf. via tandem high-speed counter-current chromatography. J. Chromatogr. B 2015, 1004, 10-16. [CrossRef] [PubMed]

17. Song, Y.L.; Wang, H.M.; Ni, F.Y.; Wang, X.J.; Zhao, Y.W.; Huang, W.Z.; Wang, Z.Z.; Xiao, W. Study on anti-inflammatory activities of phenolic acids from Lonicerae Japonicae Flos. Chin. Tradit. Herb. Drugs 2015, 46, 490-495.

18. Carnat, A.; Heitz, A.; Fraisse, D.; Carnat, A.P.; Lamaison, J.L. Major dicaffeoylquinic acids from Artemisia vulgaris. Fitoterapia 2000, 71, 587-589. [CrossRef]

19. Wang, J.; Xu, Q.L.; Zhou, Z.Y.; Tan, J.W. Caffeoylquinic acid derivatives from stems of Akebia trifoliate. J. Chin. Med. Mater. 2014, 37, 1190-1193.

20. Sun, Y.; Ma, X.B.; Liu, J.X. Compounds from fraction with cardiovascular activity of chrysanthemum indicum. China J. Chin. Mater. Med. 2012, 37, 61-65.

21. Zou, X.W.; Liu, D.; Liu, Y.P.; Fu, Y.H.; Zhang, X.Z.; Xiu, Z.L.; Xiao, H.B. Isolation and characterization of two new phenolic acids from cultured cells of Saussurea involucrate. Phytochem. Lett. 2014, 7, 133-136. [CrossRef]

22. Zhu, X.F.; Zhang, H.X.; Lo, R. Three di-O-caffeoylquinic acid derivatives from the heads of Cynara scolymus L. Nat. Prod. Res. 2009, 23, 527-532. [CrossRef] [PubMed]

Sample Availability: Not available.

(C) 2018 by the authors. Licensee MDPI, Basel, Switzerland. This article is an open access article distributed under the terms and conditions of the Creative Commons Attribution (CC BY) license (http:/ / creativecommons.org/licenses/by/4.0/). 